\title{
Localização, Segmentação e Quantificação de Placas Ateroscleróticas Calcificadas e Não Calcificadas
}

\section{Localization, Segmentation and Quantification of Calcified and Non-Calcified Atherosclerotic Plaques}

Leandro Milani Corrêa ${ }^{1}$, Bruno Clemente Guingo²

\section{RESUMO}

Este artigo propõe um método para localizar, segmentar e quantificar as formações de placas ateroscleróticas calcificadas e não calcificadas nas artérias coronárias com a aplicação de técnicas de contornos ativos geodésicos e segmentação de imagens. A aterosclerose é a principal causa de morte em pessoas com mais de 45 anos e vários fatores contribuem para aumentar o risco porque a artéria sofre uma mudança, reage multiplicando suas células e recebe o depósito de substâncias circulantes, o que promoverá seu endurecimento através do processo de calcificação. A base de dados das imagens utilizadas neste estudo consiste em 75 imagens de tomografia computadorizada e em todas as imagens foi possível quantificar as placas. $O$ algoritmo desenvolvido localiza a região de interesse, faz sua segmentação, quantificação e apresenta uma nova imagem com o tamanho da região de calcificação destacada.

Palavras-chave: Contornos ativos geodésicos. Tomografia computadorizada. Segmentação de Imagens. Placas ateroscleróticas. Artérias coronárias.

\section{ABSTRACT}

This article proposes a method for locate, segment and quantify calcified and noncalcified atherosclerotic plaque formations within coronary arteries with the application of geodesic active contours techniques and segmentation of images. Atherosclerosis is the leading cause of death in people over the age of 45 , and several factors contribute to increased risk because the artery undergoes a change, reacts by multiplying its cells and receives the deposit of circulating substances, which will promote their hardening through the calcification process. The database of images that were used in this study consists of 75 images of computed tomography and in all images was possible to quantify the plaques. The developed algorithm locates the region of interest, makes its segmentation, quantification and presents a new image with the size the highlighted calcification region.

Keywords: Geodesic active contours. Computed tomography. Image Segmentation. Atherosclerotic plaques. Coronary arteries.
Acadêmico do curso de Engenharia Mecânica da Universidade Católica de Petrópolis.

E-mail:

leandromilani@bol.com.br

2 Professor Assistente do Curso de Engenharia da Computação da Universidade Católica de Petrópolis. 


\section{INTRODUÇĀOO}

O processamento de imagens é uma área da ciência da computação que vem ganhando muito espaço, pois diversos pesquisadores vêm desenvolvendo trabalhos, principalmente voltados para encontrar regiões de interesse em imagens médicas, que possam facilitar o diagnóstico médico.

Atualmente, no Brasil, pode ser encontrado em diversos cursos de graduação e pósgraduação disciplinas que atuam no processamento de imagens, o que permitiu o aumento das investigações nessa área, a ampliação e difusão do conhecimento das técnicas aplicadas nas soluções de problemas, que antes estavam restritos aos centros de pesquisas e universidades situados na Europa, Ásia ou América do Norte.

Feijóo (2009) diz que a popularização cada vez maior do uso da internet, modernização de bancos de dados on-line, simulação de ambientes reais e o desenvolvimento de modernas técnicas de obtenção de imagens em três dimensões por meio dos computadores deverá, ao longo dos próximos anos, trazer enormes avanços na medicina. Inúmeros trabalhos estão sendo desenvolvidos, valendo destacar os resultados obtidos em modelagem e simulação do sistema cardiovascular humano, a reconstrução craniofacial e os sistemas de informações em saúde.

Nesta vertente, os investimentos alocados para trabalhos na área de computação ligados a medicina tiveram um grande aumento e houve uma divisão em quatro categorias: geração de imagens, análise de imagens, gerenciamento de imagens, e gerenciamento de informações. $O$ foco deste trabalho se dá na análise de imagens.

Desde que Röentgen, em 1895, descobriu a existência de uma radiação até então desconhecida, capaz de atravessar materiais, ser parcial ou totalmente absorvida por eles e fixar-se em anteparos, a dinâmica dos exames médicos sofreu drásticas mudanças. Com a descoberta dos Raios- $X$, há pouco mais de 100 anos, tornou-se possível a visualização de estruturas internas do corpo e diagnosticar anomalias. A partir daí, o avanço na aquisição, processamento e armazenamento de imagens médicas vem permitindo o aperfeiçoamento de diagnósticos e tratamentos de doenças de naturezas diversas. Cada modalidade de imagem médica atua de forma diferenciada. Sua aquisição visa a ressaltar as diferenças entre os diversos tipos de tecidos existentes na natureza e, especialmente, aqueles que compõem o corpo humano, fazendo com que estruturas de interesse se sobressaiam e, assim, possam ser identificadas como eventuais anomalias (NUNES, 2006). 
Maintz e Viergever (1998) lembram que as modalidades médicas podem ser classificadas em duas categorias globais: anatômicas e funcionais. As modalidades anatômicas são dedicadas a identificar morfologias, incluindo Raios- $\mathrm{X}$, Tomografia Computadorizada (TC), Ressonância Magnética Nuclear (RMN), Ultrassom (US), entre outras. Há outras modalidades derivadas dessas primeiras, sendo citadas por nomenclaturas diferentes como Angiografia por Ressonância Magnética (derivada da RMN), Angiografia por Tomografia Computadorizada (derivada da TC), Angiografia por Subtração Digital (derivada de Raios-X) e Doppler (derivada de US). As modalidades funcionais são aquelas que têm o objetivo de adquirir informação a respeito do metabolismo relacionado a uma anatomia, incluindo cintilografia, SPECT (single photon emission computed tomography), PET (positron emission tomography), modalidades de medicina nuclear, RMN funcional, entre outras.

As imagens tomográficas que foram objetos deste estudo podem ser obtidas em dois planos: axial (perpendicular ao maior eixo do corpo) e o coronal (visão frontal). A principal vantagem da TC é que permite o estudo de seções transversais do corpo vivo. É uma grande melhoria em relação às capacidades da radiografia convencional, pois permite a detecção ou o estudo de anomalias que não seria possível senão através de métodos invasivos (FARHOOD, 2010).

A aplicabilidade clínica da TC na imagem cardíaca foi limitada até pouco tempo atrás devido à sua baixa resolução espacial e temporal. Só recentemente a tecnologia da TC alcançou o nível exigido para a imagem de um órgão em constante movimento e a resolução espacial capaz de avaliar adequadamente vasos tão pequenos como as artérias coronárias. No diagnóstico de doenças cardiovasculares, a importância dos exames de imagem tem aumentado significativamente nos últimos anos. A radiologia cardiovascular tem se destacado por oferecer métodos menos invasivos e mais precisos de avaliação. Com avanços tecnológicos permanentes, os exames de imagem contribuem para um diagnóstico mais detalhado da situação do músculo cardíaco, do bombeamento de sangue e dos vasos sanguíneos, além da avaliação precisa do risco de infarto (HOCHHEGGER; CORDENONSI, 2013).

Isso é fundamental para a escolha do melhor tratamento em cada caso, bem como permite uma intervenção preventiva de maior sucesso além das tradicionais ecocardiografia e angiografia coronária com cateteres (ou cateterismo), tem aumentado a utilização de exames como a angiotomografia computadorizada (ATC) das artérias 
coronárias e a ressonância magnética cardíaca, que utilizam tecnologias modernas e oferecem mais conforto e menor risco ao paciente (DEWEY, 2009).

Além disso, as tomografias cardíacas em geral podem ser uma ferramenta útil aos cardiologistas no auxílio de diagnósticos de outras patologias ligadas ao órgão em questão, tais como as doenças cardíacas congénitas, do pericárdio e da aorta (ZUN, 2013). Não à toa, para ajudar que se extraia o máximo de informação útil de tais imagens, a angiografia é uma importante área do processamento digital de imagens, do qual se utilizam técnicas computacionais que visam ao realce da estrutura dos vasos sanguíneos a serem estudados (DUERINCKX, 2002).

Neste contexto este trabalho visa atuar em imagens de tomografia computadorizada do coração cuja finalidade é localizar, segmentar e quantificar as placas ateroscleróticas calcificadas e não calcificadas em artérias coronárias de forma automática, sem nenhuma interveniência humana.

\section{MATERIAIS E METODOS}

As imagens que fizeram parte deste estudo são oriundas de Halpern (2011), de Hochhegger e Cordenonsi (2013) e de Zun (2013).

A figura 1 apresenta um exemplo do conjunto de dados com três calcificações aparentes e com um tamanho significativo.

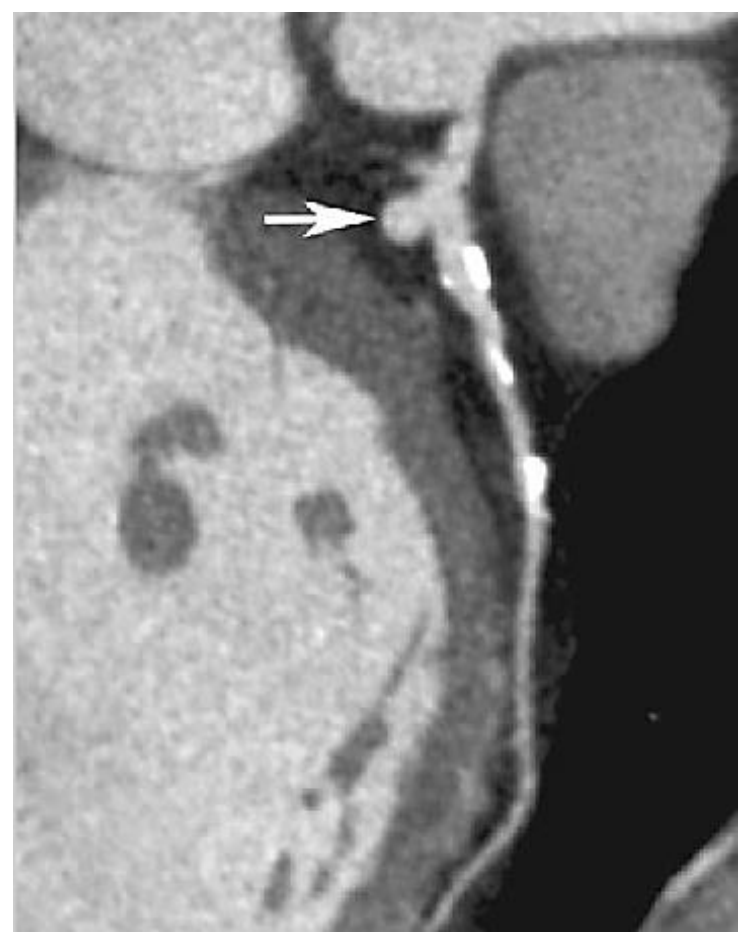

Figura 1. Imagem do conjunto de dados com três calcificações aparentes

Fonte: Halpern, 2011. 
A figura 2 mostra outro exemplo do conjunto de dados com várias calcificações (sete), sendo que todas possuem tamanhos diferentes, desde um valor pequeno até ao tamanho da espessura da própria artéria.

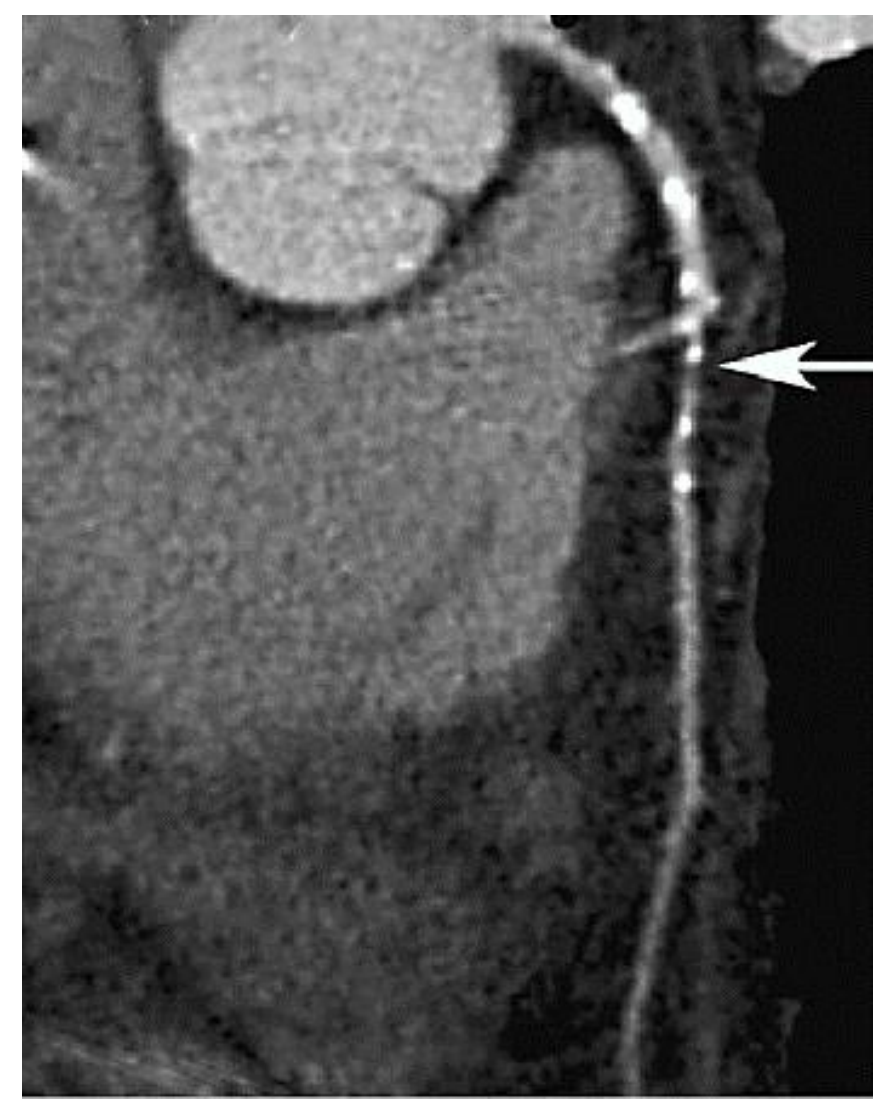

Figura 2. Imagem do conjunto de dados com várias calcificações aparentes Fonte: Halpern, 2011.

A técnica de contornos ativos geodésicos, que foi desenvolvida baseada nos conceitos introduzidos por Kass et al (1987), cuja ideia geral do modelo é a utilização de uma curva de minimização de energia para extrair características importantes de uma imagem. Essa energia associada à curva é definida de forma a que ela seja mínima quando a curva se encontre sobre uma região com as características que se deseja extrair, dessa forma a função de energia passa a funcionar como uma função objetivo.

A localização e segmentação da região com as calcificações foram realizadas aplicando-se o método do Otsu (1979), que faz a binarização da imagem, e posteriormente, com a aplicação de um processo baseado no filtro de Gabor (ANGELO, 2000). 
Para a quantificação o algoritmo verifica a quantidade de pixels presentes na imagem via uma varredura, faz a quantificação em número de pixels de cada área destacada como calcificada no processo de segmentação.

Todo o desenvolvimento computacional se deu no MATLAB® R2010a.

\section{RESULTADOS}

O processamento da imagem, para localizar e segmentar as artérias coronárias, consistiu, inicialmente, em binarizar a imagem, aplicando-se o método do OTSU (OTSU, 1979) para realizar a limiarização e reduzir os pixels da imagem que tivessem valor zero com vizinhos de valores 1, e posteriormente, aplicar o algoritmo baseado no filtro de Gabor (ANGELO, 2000).

Estudos verificam que as análises por técnicas de Fourier não revelam distribuições locais, pois apenas apresentam as frequências espaciais presentes na imagem original, não identificando a sua posição na mesma. O filtro de Gabor por sua vez permite selecionar feições cujas características ficam determinadas por frequências espaciais diretamente no domínio espacial, sem a necessidade de ter que se trabalhar no domínio das frequências (SANTOS; ANGELO, 2007).

A Figura 3 é outro exemplo de amostra da base de dados e a Figura 4 exemplifica o processamento do contorno ativo geodésico aplicado na Figura 3.

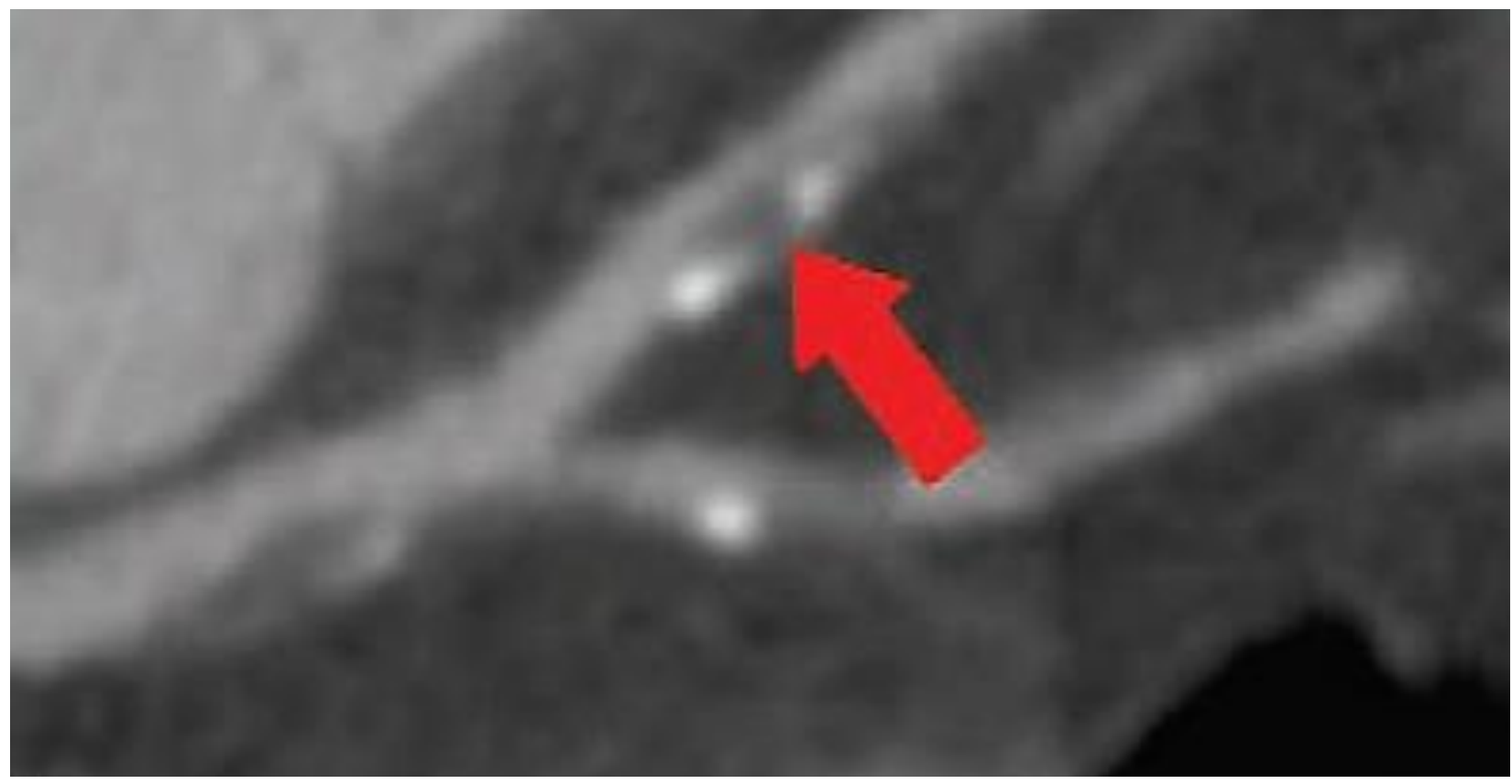

Figura 3. Imagem do conjunto de dados

Fonte: Hochhegger; Cordenonsi, 2013. 
Corrêa, L. M., Guingo, B. C.

Localização, Segmentação e Quantificação de Placas Ateroscleróticas Calcificadas e Não Calcificadas.

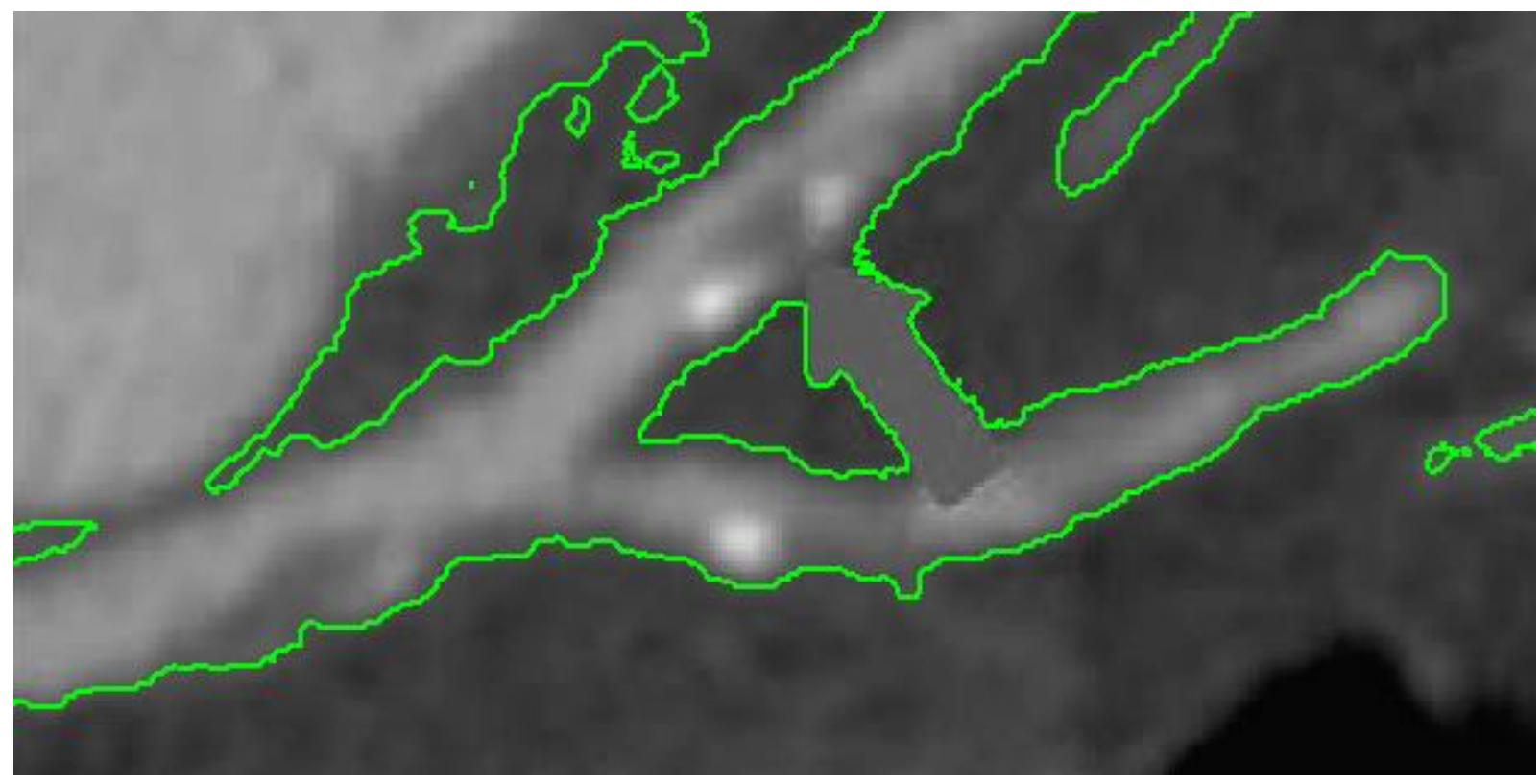

Figura 4. Imagem Processada com contorno ativo geodésico

Fonte: Hochhegger; Cordenonsi, 2013, alterada pelo autor.

É exemplificado na figura 5, o processamento do contorno ativo geodésico realizado na figura 2 (original).

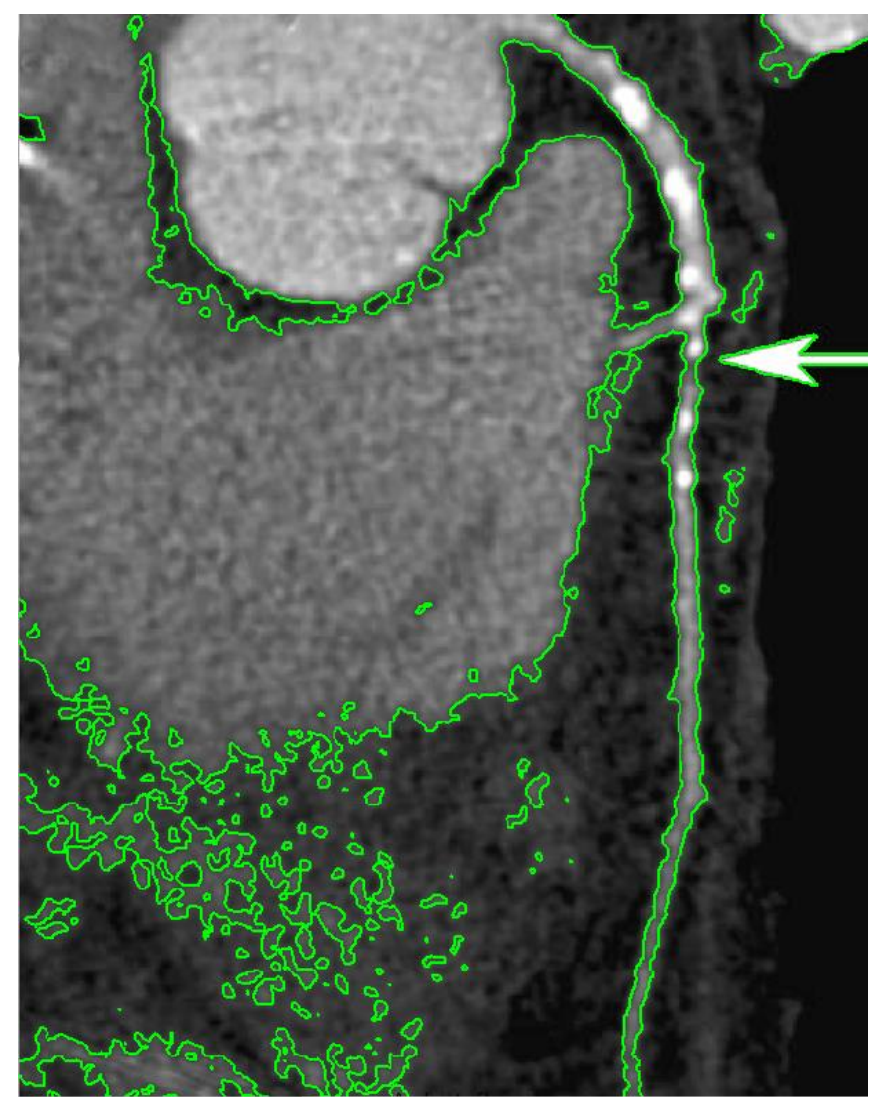

Figura 5. Imagem Processada com contorno ativo geodésico

Fonte: Halpern, 2011, alterada pelo autor. 
A figura 6 ilustra o processamento da segmentação das regiões com formações ateroscleróticas realizado na figura 5.

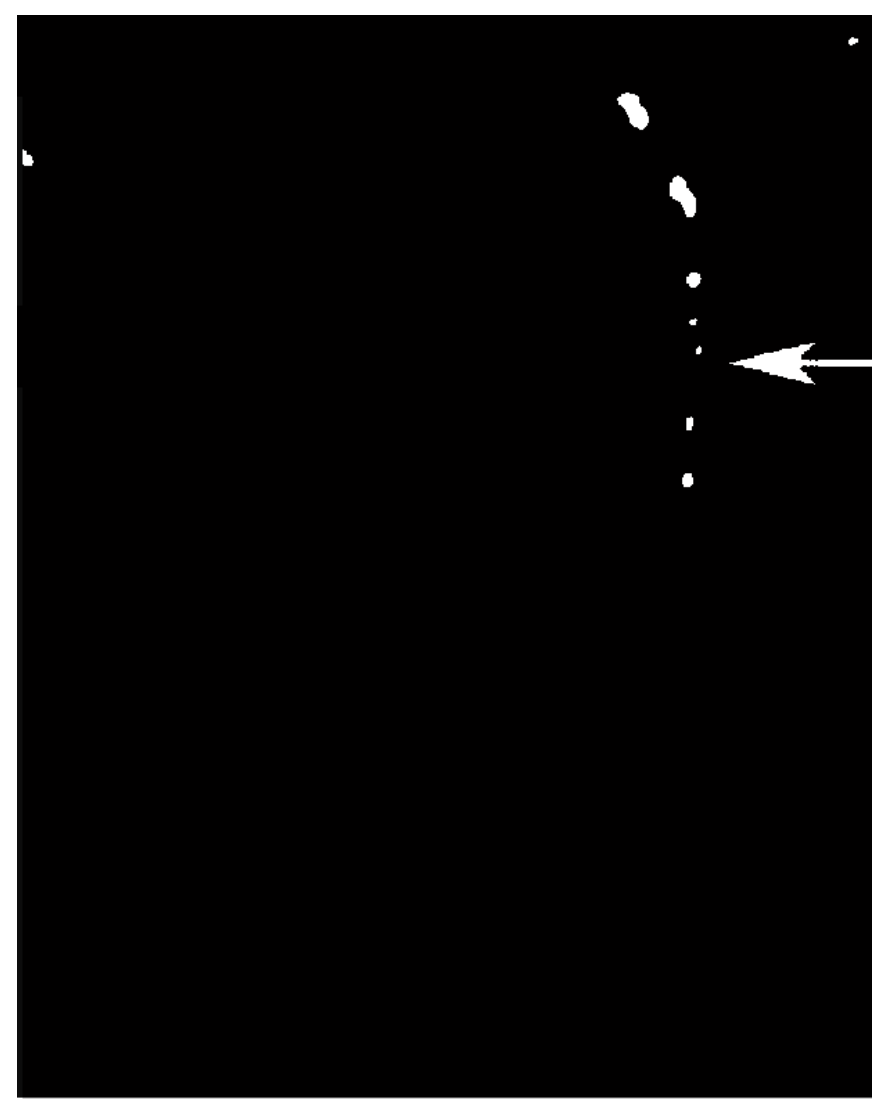

Figura 6. Segmentação de formações ateroscleróticas

Fonte: Halpern, 2011, alterada pelo autor.

O processamento final do algoritmo é apresentado na figura 7 e pode-se verificar que as regiões de interesse (formações ateroscleróticas) que foram segmentadas na etapa anterior tem sua quantificação realizada.

Os valores obtidos para as três maiores regiões foram: 347, 341, 89 e para a menor região foi de 29 pixels. 


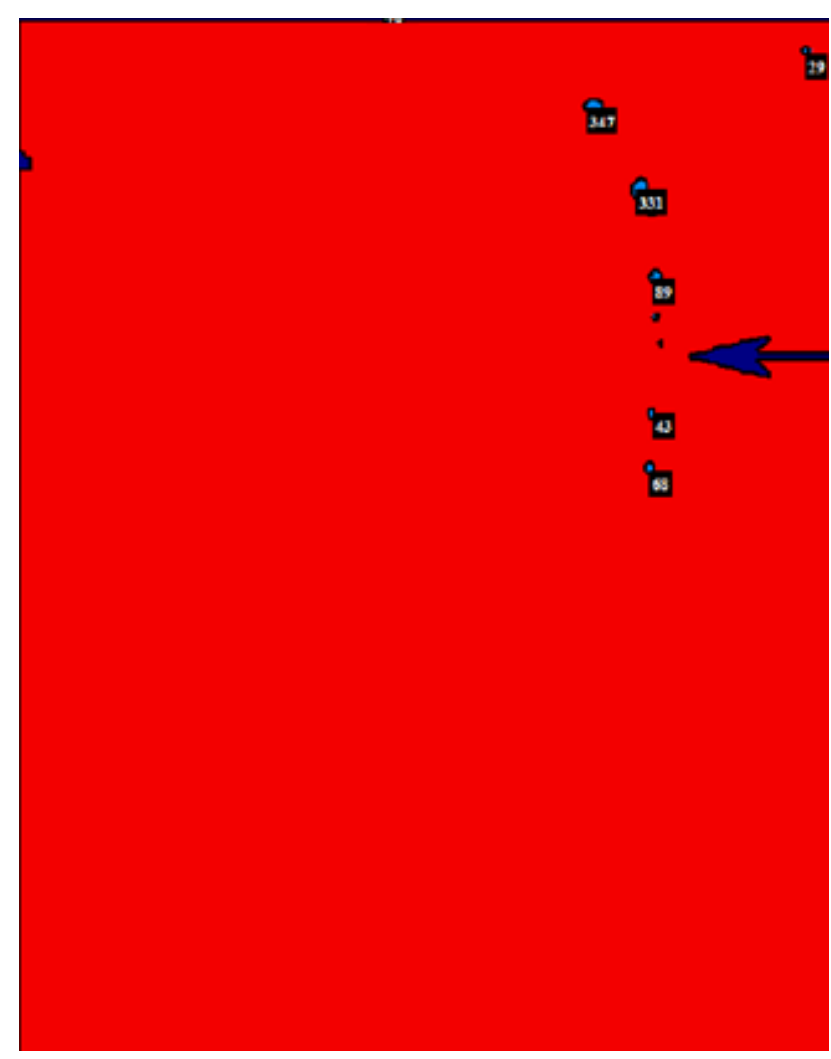

Figura 7. Quantificação das formações ateroscleróticas

Fonte: Halpern, 2011, alterada pelo autor.

\section{DISCUSSAOO}

Referente à segmentação a publicação de Jodas et al (2017) diz que para segmentar as placas ateroscleróticas e os principais componentes relacionados nas imagens de RM da artéria carótida utiliza o algoritmo K-means, que faz a obtenção das regiões compostas pelos pixels de baixa intensidade e relacionados ao lúmen e o fundo da imagem em análise. Uma vez que a região do lúmen é aproximadamente circular, os índices de circularidade e irregularidade são usados para encontrar a região desejada entre as várias candidatas de região. Para a determinação final das regiões com as placas ateroscleróticas faz uso do algoritmo de C-means fuzzy.

Os trabalhos desenvolvidos em Bonanno et al (2017) e em Sharma et al (2006) fazem uso de contorno ativo geodésico para realizar a segmentação das placas ateroscleróticas.

Em Loizou et al (2007) foram avaliados quatro sistemas de segmentação: William e Shah, Balão, Lai e Chin, e GVF. Os melhores resultados foram obtidos com uso de contorno ativo geodésico em Lai e Chin. 
O trabalho desenvolvido por Weert et al (2008), que visa avaliar o volume da placa aterosclerótica faz uso de uma ferramenta manual, deixando a tarefa de marcação da região de interesse ao usuário. Já a publicação de Sillesen et al (2012) fez uso do Philips QLAB Quantification Software, um programa comercial que realiza de forma semiautomática a quantificação das placas ateroscleróticas e em Brodoefel et al (2009) fez uso da ferramenta comercial Volcano para o processamento das imagens. Em nenhum destes trabalhos é explicado como é feita a quantificação, apenas é informado que realizam.

\section{CONSIDERAÇOES FINAIS}

O método proposto efetuou de forma muito satisfatória a localização, a segmentação e a quantificação das regiões de interesse (formações ateroscleróticas) no conjunto de imagens, mesmo em regiões de tamanho extremamente pequeno (abaixo de 60 pixels). Entretanto, cabe ainda aperfeiçoamentos no algoritmo no que diz respeito a realizar a transformação da quantificação de pixels para milímetros ou outra unidade de medida. Para confirmação da eficiência do algoritmo, faz-se necessário uma nova análise numa base de dados com maior número de amostras.

Vale ressaltar que o algoritmo atua desde a imagem original até a imagem final processada, que apresenta a quantificação das regiões com as formações ateroscleróticas sem nenhuma interferência humana.

\section{REFERÉNCIAS}

ANGELO, N. P. Aplicação de Filtros de Gabor no processo de classificação de imagens digitais com base em atributos de textura. Dissertação (Mestrado). Universidade Federal do Rio Grande do Sul, Porto Alegre, 2000.

BONANNO, L.; SOTTILE, F.; CIURLEO, R.; DI LORENZO, G.; BRUSCHETTA, D.; BRAMANTI, A.; ASCENTI, G.; BRAMANTI, P.; MARINO, S. Automatic Algorithm for Segmentation of Atherosclerotic Carotid Plaque. J Stroke Cerebrovasc Dis.;26(2):411416, 2017

BRODOEFEL, H.; BURGSTAHLER, C.; SABIR, A.; YAM, C. S.; KHOSA, F.; CLAUSSEN, C. D.; CLOUSE, M. E. Coronary Plaque Quantification by Voxel Analysis - DualSource MDCT Angiography Versus Intravascular Sonography. AJR: 192, 2009.

DEWEY, M. Coronary CT Angiography. Nova York: Springer-Verlag, 2009.

DUERINCKX, A. J. Coronary magnetic resonance angiography. Nova York: SpringerVerlag, 2002. 
FARHOOD, S. A. Computed-Tomography-Based Atlas and Reference. WileyBlackwell, 2010.

FEIJÓO R. A. Uso de computadores contribui para avanço da medicina no país [internet]. 2017; Disponível a partir de: http://www.faperj.br/ ?id=1423.2.6. Acesso em: 26/07/2017.

HALPERN, E. J. Clinical Cardiac CT: Anatomy and Function. 2 $2^{\mathrm{a}}$ Edição. Nova York, 2011.

HOCHHEGGER. B. ; CORDENONSI, I. C. O. Tomografia Computadorizada Cardíaca: O que nós devemos saber. Revista da AMRIGS, Porto Alegre, 2013.

JODAS, D. S.; PEREIRA, A. S.; TAVARES, J. M. R. S. Segmentation of atherosclerotic plaques in MR carotid artery images. 14th U.S. National Congress on Computational Mechanics (USNCCM14), Palais des Congrès de Montréal, Québec, Canada, July 17-20, 2017.

KASS, M., WITKIN, A., TERZOPOULOS, D. Snakes: Active contour models. International Journal of Computer Vision, v. 1, n. 4, p. 321-331, 1987.

LOIZOU, C. P.; PATTICHIS, C. S.; PANTZIARIS, M.; NICOLAIDES, A. An integrated system for the segmentation of atherosclerosis carotid plaque. IEEE Trans Inform Technol Biomed, 11:661-667, 2007.

MAINTZ, J. B. A.; VIERGEVER, M. A. A survey of medical image registration. Medical Image Analysis, v. 2, n. 1, p.1-36, 1998.

NUNES, F. L. S. Introdução ao Processamento de Imagens Médicas para Auxílio ao Diagnóstico - Uma Visão Prática. In: Breitman, K. e Anido, R. (Org). Atualizações em Informática, v. 1, p.73126,2006.

OTSU, N. A Threshold Selection Method from Gray-Level Histograms, IEEE Transactions on Systems, Man, and Cybernetics, 1979.

SANTOS, M. S.; ANGELO, N. P. Aplicação do Filtro de Gabor Associado ao Classificador de Máxima Verossimilhança Gaussiana na Segmentação de Imagens Baseadas em Características Texturais. Revista Hifen, v. 1, n. 59, 2007.

SHARMA, R. et al. Segmentation Methods in Atherosclerosis Vascular Imaging. Informatica Médica Slovenica, 2006.

SILLESEN, $H$. et al. Carotid Plaque Burden as a Measure of Subclinical Atherosclerosis Comparison with Other Tests for Subclinical Arterial Disease in the High Risk Plaque Biolmage Study. JACC: Cardiovascular Imaging, vol. 5, número 7, 2012.

SUN, Z. Coronary Computed Tomography Angiography in Coronary Artery Disease: a Systematic Review of Image Quality, Diagnostic Accuracy and Radiation Dose. Nova York, 2013.

WEERT, T. T. et al. Assessment of atherosclerotic carotid plaque volume with multidetector computed tomography angiography. Int J Cardiovasc Imaging, 24:751759, 2008. 